\title{
28 Research Square \\ Species distribution of yeasts recovered from different clinical samples Addiss Ababa,Ethiopia
}

Elias Seyoum Deribe ( $\nabla$ eliasseyoum2@gmail.com )

Ethiopian Public Health Institute

Adane Bitew

Ethiopian Public Health Institute

Amete Mihret

Ethiopian Public Health Institute

Research article

Keywords: Candida albicans, Non- albican candida species, Candidasis

Posted Date: April 17th, 2019

DOI: https://doi.org/10.21203/rs.2.9212/v1

License: (1) This work is licensed under a Creative Commons Attribution 4.0 International License.

Read Full License 


\section{Abstract}

Background Currently, fungal diseases of humans are among the most problematic illnesses is the result of an increase in opportunistic fungal infections caused by rise in the number of immune-compromised patients. Particularly, yeasts are the most common cause of fungal infections leading to a range superficial type to life-threatening invasive infections. The aim of this study is to determine species distribution of yeasts particularly of Candida species recovered from different clinical samples. Method A cross sectional study was conducted from January 01, 2018 -September 30, 2018 in Addis Ababa, Arsho laboratory. Oropharengial swabs, nail scrapings, sputum, corneal scrapings, and vaginal swabs were collected from patients with signs and symptoms of infections. Identification and antifungal susceptibility testing was performed by using YST card through VITEK 2 compact system. Data was entered and analyzed using SPSS version 20. Result A total of 209 yeasts were recovered of which $49.8 \%$ were Candida albicans, $43.1 \%$ were non-albicans candida species and $7.2 \%$ were yeasts other than Candida species. The current study, eighteen (18) different types of yeast species were identified from different clinical samples. All recognized yeast considered as a causative agent of fungal infection in previously reported data in worldwide. Conclusion C. albicans remaining the predominant species, but the emergence of non-albicans Candida and other than candida species have increased. Thus, more studies on Candida prevalence are needed throughout country.

\section{Back Ground}

Currently, fungal diseases of humans are among the most problematic illnesses to manage. Some fungi cause disease in immune-competent persons (fungal true pathogens), but most fungal infections occur in immune-compromised individuals (opportunistic fungal pathogens). Since risk-factors for opportunistic fungal infections continue to increase in frequency, it is likely that the incidence of opportunistic fungal infections will continue to increase in the future. An increase in opportunistic fungal infections are the result of an increase in the number of immune-compromised patients, thereby threaten the achievement of the newest medical advances in cancer care, solid organ and hematopoietic stem cell transplantation, neonatal medicine, autoimmune disease therapies, trauma and intensive care, and sophisticated surgery [1-4].

Fungi can cause infections ranging from easily treatable superficial type to life-threatening invasive infections and it has the capability to infect humans of all age groups [12]. Particularly, yeasts are the most common cause of fungal infections, leading to a range of life-threatening invasive diseases such as blood stream candidiasis, pneumonia, and cryptococcal meningitis to non-life-threatening mucocutaneous candidiasis such as genitourinary candidiasis, vulvovaginal candidiasis, and oropharyngeal candidiasis [5]. They are also important cause of superficial mycosis such as onychomycosis [27]. Among fungal infections, blood stream candida infection (candidemia) and cryptococcosis are commonly associated with high morbidity and mortality rate. For example, Candida species are among the top ten pathogens causing bloodstream infections resulting in significant increase in the length of patients' hospitalization and in healthcare costs [5]. 
Currently, there are more than 150 known species of Candida [3], and more than 17 different Candida spp. are known as etiological agents of human infection [13].Among candida, Candida albicans is the most common infectious agent. However, there has been an important shift to non-albicans Candida species.

The aim of this study was to determine distribution profile of yeasts particularly of Candida species recovered from different clinical samples collected from patients referred for culture testing by using the VITEK 2 compact system.

\section{Methods}

\subsection{Study area, Study design and Period}

The study was conducted at Arsho Medical laboratory, Addis Ababa, Ethiopia. Arsho is a brand of private diagnostic Laboratory practice in Ethiopia. It originated in 1972 in a small individual practice and grows up to internationally accredited big service and name. Their personnel specializing in a wide variety of disciplines including microbiology work to ensure best quality and quick delivery of medical result. Daily patient flow for microbiology diagnosis approximately ten patients per day.A cross sectional study was conducted from January 01, 2018 -September 30, 2018.

Sample collection

All samples were collected according to standardized operating procedures. Clinical samples including oropharengial swab, nail scrapings, sputum, corneal scrapings, and vaginal swabs were collected from patients with signs and symptoms of infections and referred to the study site for culture and susceptibility testing.A portion of each clinical specimen was inoculated onto bacteriological culture media for routine activity and incubated at appropriate temperature and period according to standard protocols related to each sample. The other portion of each clinical samples were inoculated on to Sabouraud dextrose agar (Oxoid, Basingstoke,UK) to which $50 \mu \mathrm{g} / 1 \mathrm{ml}$ gentamicin is incorporated. Inoculated sample were kept at least 72 hours a temperature of $370 \mathrm{C}$. Yeast isolates were transferred to tryptic soya broth with $20 \%$ glycerol and transport to the Ethiopian public health institute national reference laboratory of clinical bacteriology and mycology case team and stored at minus $80 \mathrm{o}$ Refrigerator until used.

\section{Identification:}

Identification testing was performed by YST card through VITEK 2 system.

Compared the performance of the VITEK 2 YST identification card to the RapID Yeast Plus system using 750 clinical yeast isolates, using 16S rRNA sequence analysis was used as the reference method ,98.2\% of isolates were correctly identified to the species level by the VITEK 2 system [14]. 
The purity and viability of yeast original cultures were checked by plating yeast colonies on Sabouraud dextrose agar (Oxoid, Basingstoke,UK). Yeast identification testing were determined by the automated VITEK 2 compact system (bioMérieux, France) using YST-21343. The inoculums suspensions for the VITEK 2 were prepared in sterile saline at a turbidity equal to a $2.0 \mathrm{McFarland}$ standard, as measured using a Densichek instrument (bioMérieux).The YST-21343 were automatically filled with the prepared culture suspension, sealed, and incubated by the VITEK 2 instrument. The cards were incubated at 35.5 ${ }^{\circ} \mathrm{C}$ for $18 \mathrm{~h}$, and data were collected at 15 -min intervals during the entire incubation period and final identification testing results were obtained in approximately $18 \mathrm{~h}$ or less. The final profile results were compared with the database, and the identification of the unknown organism was obtained. A final identification of excellent, very good, good, acceptable, or low- discrimination was considered to be correct.

\section{Quality Assurance:}

Expiry date of media, VITEK card of YST, sterility and performance of media and VITEK card checked and a standard protocol was followed. Specimens were collected following SOPs and processed in Arsho microbiology laboratory. Isolates were transported to the laboratory of Ethiopian public health institution national reference laboratory of clinical bacteriology and mycology as soon as possible after identify isolates for further identification test. Pre-test was done before regular data collection was started. For performance check VITEK 2 Compact system tested by using Standard strains of Candida albicans ATCC 10231 before any isolate tested. Reference strains for quality control were passed. Quality of the data were maintained by coding the isolates with unique number and finally all clinical isolates were preserved in deep freeze using $20 \%$ glycerol with trypticase soya broth (TSY) in case needed or for future further investigation. Data were checked for completeness before analysis.

\section{Data Processing and Analysis}

Results were compiled and entered in to SPSS version 20 Software and were analyzed to determine frequency, percentage and compare distribution of each etiologic agent by anatomic site of infection involved.

\section{Ethical Consideration}

All ethical considerations and obligations were duly addressed, and the study was conducted after the approval of the department of research and ethical review committee (DRERC) of the department of Medical Laboratory Sciences. Written informed consent was obtained from the participants before data collection. Each respondent was given the right to refuse to take part in the study and to withdraw at any time during the study period. All the information obtained from the study subjects were coded to maintain confidentially. When the participants are found to be positive for fungal pathogen, they were informed to 
the hospital clinician and received proper treatment. An assent form was completed and was signed by a family member and/or adult guardian for participants under the age of less than 18 years.

\section{Results}

As shown in Table 1, a total of 209 yeasts were recovered of which 49.8\% (104/209) were Candida albicans, 43.1\% (90/209) were non-albicans candida species and 7.2\% (15/209) were yeasts other than Candida species. The two hundred nine isolated from different body sites which consisted of $24 \%$ (50/209) oropharengial, $41 \%$ (87/209) vaginal, 12\% (25/209) nail 5.7\% (12/209) eye discharges and 17 $\%(35 / 209)$ sputum were collected and evaluated for yeast species distributions. Yeast species with major candida species identified in different quantity of distribution. The most commonly isolated species were: Candida albicans 49.8\% (104/209) followed by Candida krusie $6.7 \%$ (14/209), Candida famata 6.2\% (13/209), each of Creptococcus laurenti and Candida rugosa $4.8 \%(10 / 209)$,Candida Iusitaniae 4.3\% (9/209), each of Candida parapsilosis, Candida kefyr,Candida lipolytica and Candida ciferrii 2.9\% (6/209), Candida guilliermondii 2.4\% (5/209),Candida dubliniensis $3.3 \%$ (7/209), each of Cryptococcus neoformans and Candida pelliculosa 1.9\% (4/209) and other yeast species 2.4\% (5/209).

The proportion of $C$. albicans to non- albicans candida species was 1.2 to 1 .C. krusei $15.6 \%$ (14/90), C. famata $14.4 \%$ (13/90), C. rugosa $11.1 \%$ (10/90), and C. Iusitaniae $10.0 \%$ (9/90) were the commonest isolates among non-albican candidia species. Candida krusi the main isolated in vaginal isolate while Candida rugosa and Candida famata from sputum and nail specimen, respectively. Whereas, yeast fungal infections of the eye showing highest variability of non-albican candida species with equal frequency (Table 1). Among yeasts other than Candida species Cryptococcus laurenti represented $66.7 \%$ $(10 / 15)$ this group of yeasts.

Table 1. Species distribution of yeasts isolated from different clinical samples from January 2018 to September 2018 in Addis Ababa, Ethiopia. 


$\begin{array}{lllllll}\text { Species } & \begin{array}{l}\text { Vaginal } \\ \text { Discharge }\end{array} & \text { Oropharyngeal } & \text { Nail } & \begin{array}{l}\text { Eye } \\ \text { Discharge }\end{array} & \text { Sputum } & \text { Total } \\ \text { Candida albicans } & 52 & 28 & 4 & 5 & 15 & 104 \\ \text { Sub-total } & & 104 & & & & \end{array}$

Non-albicans candida

\begin{tabular}{lllllll} 
C. krusie & 11 & 0 & 0 & 2 & 1 & 14 \\
C.famata & 2 & 4 & 5 & 1 & 1 & 13 \\
\hline C. guilliermondii & 0 & 3 & 1 & 1 & 0 & 5 \\
\hline C. lipolytica & 2 & 4 & 0 & 0 & 0 & 6 \\
\hline C.pelliculosa & 1 & 0 & 3 & 0 & 0 & 4 \\
\hline C. intermedia & 0 & 0 & 1 & 0 & 0 & 1 \\
\hline C. utilis & 0 & 1 & 0 & 0 & 0 & 1 \\
\hline C.rugosa & 0 & 2 & 1 & 1 & 6 & 10 \\
\hline C. glabrata & 2 & 0 & 0 & 0 & 0 & 2 \\
\hline C. lusitaniae & 3 & 2 & 3 & 1 & 0 & 9 \\
\hline C. kefyr & 1 & 2 & 1 & 1 & 1 & 6 \\
C. dubliniensis & 5 & 0 & 0 & 0 & 2 & 7 \\
\hline C. parapsilosi & 2 & 4 & 0 & 0 & 0 & 6 \\
\hline C. ciferrii & 2 & 0 & 0 & 0 & 4 & 6
\end{tabular}

\section{Sub total}

90

\section{Other than Candida}

species yeast

$\begin{array}{lllllll}\text { Cryptoccocus laurenti } & 3 & 0 & 4 & 0 & 3 & 10 \\ \text { C. neoformans } & 1 & 0 & 1 & 0 & 2 & 4 \\ \text { Trichosporn mucoides } & 0 & 0 & 1 & 0 & 0 & 1 \\ \text { Sub-total } & 15 & 25 & 12 & 35 & 209 \\ \text { Grand Total } & 87 & 50 & & & & \end{array}$

Figure I: Frequency of Candida albican, non-albicans Candida and other yeasts species from different clinical samples at Arsho medical laboratory in Addis Ababa, Ethiopia from January, 2018 to September. 


\section{Discussion}

The incidence of opportunistic fungal infections such as Candida infections has considerably increased recently. Given the fact that many clinical microbiology laboratories in Ethiopia do not perform identification of yeasts beyond direct microscopy, accurate identification of Candida down to the species level is crucial. This is because different species have different antifungal susceptibility profile and the incidence of non-albicans candida is increasing. In the present study, identification of yeasts down to the species level profile were determined in an automated manner by the VITEK 2 compact system that are reproducible and precise when compared with other reference methods $[6,7$, and 8$]$.

Although the relative prevalence of the yeast species depends on the geographical location, patient population, and clinical settings [65], with a rise in the immunosuppressive patients, increased number of fungal infections has been reported worldwide. Simultaneously, the profile of human yeast pathogens has also been increasing [17]. Whereas, In Ethiopia little is known regarding the distribution and the in vitro antifungal susceptibility profile of yeasts isolated from patients. The different types of yeast species were identified from clinical suspect volvovaginal, oropharngial, sputum, nail and eye fungal infection patient with major Candida species of yeast. These are Candida albicans, Candida glabrata, Candida parapsilosis, Candida krusei, Candida guilliermondii, Candida lusitaniae, Candida dubliniensis, Candida pelliculosa, Candida kefyr, Candida intermedia, Candida lipolytica, Candida famata, Candida rugosa, and Candida pelliculosa, Creptococus laurentii , Creptococus neoformans, Trichosporn mucoides, Candida ciferrii. This result demonstrated similar finding with previously published data, which reported considered as a causative agent of fungal infection, in worldwide $[3,13]$. There is no recognized information regarding the profile of yeast species from Ethiopian patient s similar result with this data.

The present study, $49.8 \%$ were $C$. albicans, $43.1 \%$ were non-albicans candida species, and $7.1 \%$ were yeasts other than Candida species; the ratio between $C$. albicans to non-albicans Candida species was being1.2 to 1. Among non- albicans Candida spp. $C$. krusei (14), C. famata (13), C. rugosa (10) and $C$. lusitanitae (9) were the predominant species. C. albicans and C. krusei as the 1st and the 2nd predominate species were similar with a study conducted in Ethiopia [9] but, $C$. famata and $C$. rugosa two of the dominant species in the current study were not recovered at all by previous Ethiopian study [9], as well as in the world consider as rare finding of human pathogen [3]. Occurrence rate of Candida rugosa $4.8 \%(10 / 209)$ isolated from sputum isolate followed by oropharengial, coneal discharge and nail. These data result compare to Ghana isolates reported $0.7 \%$ of total Candida isolates (4/600), which is similar to the reported incidence of $0.6 \%$ worldwide. The variation arise may be due to regional and patient population of in this study [19] .Clinically, species of the $C$. rugosa complex have been isolated from a range of sources including blood, urine, sputum, and swabs from different anatomical sites [20]. Members of the Candida rugosa species complex have been described as emerging fungal pathogens and are responsible for a growing number of Candida infections [21].Studies are needed to better clarify the frequency of Candida rugosa infections in Ethiopian patients.

A total of 50 yeast isolates from oropharngial specimen with predominant Candida albican $56 \%(28 / 58)$ isolated. It has great variation previously reported data in Ethiopia 81\% [18] and Ghana $76.19 \%$ from HIV 
patients[15].However, one study in China from patients in intensive care units (ICU) result comparable Candida albican (40.1\%) to this finding [11]. This deviation possibly arises from the study population difference and low number of sample size. In oropharengial, the incidence rate of non-albican candida yeasts were around $44 \%(22 / 50)$ and other yeast was not found which is similar with previously study $[18,10]$.

$5.7 \%(12 / 209)$ from total of isolate recovered from eye discharge with main isolate of Candida albican and high variability of non-albican candida species with equal frequency of each $0.5 \%(1 / 209)$ that were C. Iusitaniae,C. kefyr,C. rugosa,C. famata,C. krusi and $C$. guilliermondii. Fungal keratitis is one of the most challenging types of infectious keratitis which has been gradually increasing during the past few decades. It now accounts for approximately $50 \%$ of infectious corneal diseases [22].

In the current study, the occurrence rate of non candida yeast isolates were $7.1 \%$ with highest frequency of Creptococcus laurenti $4.8 \%$ (10/209) followed by Creptococcus neoformans 2\% (4/209) and Trichosporn mucoides 0.5\%(1/209). Cryptococcus laurenti and Trichosporn mucoides are a rare human pathogen, but Creptococcus neoformans as typical fungal pathogen to immunocompromised [13]. When comparing these data of Creptococcus laurenti $4.8 \%$ (10/209) to previously one study showed $0.6 \%$ $(1 / 155)$ recovered rate in Ethiopia from orophrengial [23].Other than Cryptococcus neoformans species have classically been considered to be non-pathogenic. However, Cryptococcus albidus, Cryptococcus laurentii , Cryptococcus luteolus ,Cryptococcus uniguttulatus, Cryptococcus curvatus, have emerged as opportunistic pathogens over the last few years[24]. They have been described as opportunistic pathogens in HIV positive individuals, as well as in patients with other predisposing factors [25]. Creptococcus laurentii has been implicated in 18 cases of opportunistic infection, predominantly of the skin, bloodstream, and central nervous system. Within the non-neoformans Cryptococcal species, Cryptococcal laurentii and Cryptococcal albidus account for $80 \%$ of pathogenic infections. Although $C$. laurentii is found worldwide and its natural habitat remains largely unknown $[17,26]$, the occurrence rate $(4.8 \%)$ of its current finding is higher.

Trichosporon spp are yeast-like fungi found in soil and water. Furthermore, they belong to the normal flora of the human skin and gastrointestinal tract. The first case of onychomycosis caused by Trichosporon mucoides was published in 2011 by Malini A et al [27]. An additional cases were published in 2015 and 2016 by Capoor et al and Rizzitelli et al [28]. The published data showed Trichosporon mucoides a clinical significant isolate that is consider as human pathogen even if the occurrence of this fungi rare. This fungus is only one (1/25) Trichosporon mucoides recovered from nail that is agreeing from previously finding. However, clinical history of this patient was not evaluated. All epidemiological papers of fungal diseases in Ethiopia were reviewed. Where there was no Ethiopia data align with this finding .Perhaps, by reason of these diseases are often understudied.

Generally, this is the first report including other than candida species in Ethiopia demonstrated as species level by this amount and variance of yeast species. This explains incidence of $C$. albicans has decreased, while that of the non-albicans Candida and other yeast species has increased. Although the reason for 
the emergence of non-albicans Candida and other than candida species in large proportion in this study is not clear, the precision of the identification method (VITEK 2 compact system) and use of antifungal drugs for prophylaxis and treatment empirically in Ethiopia could be possible explanations.

\section{Conclusion}

A total of eighteen (18) different type species of yeast were isolated with $C$. albicans remaining the predominant species in all specimen type. The emergence of non-albicans Candida and other than candida species yeast have increased .Importantly, knowledge of the species of pathogenic yeast is a useful guide to the probable pattern of susceptibility and for successful treatment of patients. Therefore, epidemiological studies are required to determine the exact incidence and prevalence of these infections in a country trends over time. More studies on Candida prevalence are needed throughout country.

\section{Declarations}

I would like to acknowledge Department of Medical Laboratory Sciences, College of Health Sciences, and Addis Ababa University for giving me the opportunity to go through and develop this research. I would like to acknowledge Ethiopian Public Health Institute Directorate of parasitic, bacterial \& Zoonotic related disease unit of clinical bacteriology and mycology research case team for the primary permission to conduct this research in bacteriology laboratory using the required equipment, supplies and reagents and also I would like to extend my deepest gratitude and thanks to Dr. Adane Bitew (PhD) for his unreserved support, provision of valuable comments.

Availability of data and materials

All the data are found in the manuscript and there are no supplementary

files. The original data supporting this finding are available from the author

and can be obtained at any time upon reasonable request.

Authors' contributions

Elias seyoum(ES) has participated in culture media preparation, identification of yeasts

Pathogens, in drafting the manuscript, have given final approval of the

version to be published; analysis and interpretation of data, and agree to be

accountable for all aspects of the work in ensuring that questions related to

the accuracy or integrity of any part of the work are appropriately investigated and resolved. 
Adane bitew (AB) has participated in design, analysis and interpretation of data, yeast identification, write up of the manuscript, agree to be accountable for all

aspects of the work in ensuring that questions related to the accuracy or

Integrity of any part of the work is appropriately investigated and resolved.

Amete mihret(AM) also has participated in write up of the manuscript.

All authors approved the final version of the manuscript.

Authors' information

ES, is a medical laboratory technologist with Msc degree in microbiology,

He has been working as associate researcher and quality officer in Ethiopian Public Health Institute Directorate of parasitic, bacterial \& Zoonotic related disease unit of clinical bacteriology and mycology research case team for years. He has published by co-authors

five original articles in peer-reviewed four local and one international journal.

$A B$ is an associate professor of microbiology and consultant health science

Specialist with a PhD degree. He has been working as a researcher and

instructor offering courses to graduate students in the college of health

sciences, Addis Ababa University for more than 24 years. He has published

many original articles in peer-reviewed international journal.

AM is also a is a medical laboratory technologist with Msc degree in microbiology and and currently a PHD candidate for medical microbiologist as well as a young ambassador of American Society for microbiology as Ethiopian country representative.She is working researcher in Ethiopian Public Health Institute Directorate of parasitic, bacterial \& Zoonotic related disease unit of clinical bacteriology and mycology research case team for years. She had published by authors and co-authors original articles in peer-reviewed local and international journal.

Ethics approval and consent to participate

The study was conducted after it was ethically reviewed and approved by the Ethical Review board of the Department of Medical Laboratory Sciences (DRERC), School of Health Sciences, and Addis Ababa University. Written 
Consents were also obtained from participants. Assent form was completed

and signed by family member and/or adult guardian for participants under

the age of 18 years.

Competing interests

The authors declare that they have no competing interests.

Author details

1. Ethiopian Public Health Institute, P.O.Box:1242,Addiss Ababa,Ethiopia

*Correspondingauthor:eliasseyoum2@gmail.com,Tel:+251-912969651

2. Department of Medical Laboratory Sciences, College of Health Sciences,

Addis Ababa University, P.O. Box1176, Addis Ababa, Ethiopia.

3. Ethiopian Public Health Institute, P.O.Box:1242,Addiss Ababa,Ethiopia Reference

1. Upton A, Marr KA. Emergence of opportunistic mould infections in the hematopoietic stem cell transplant patient. Current infectious disease reports. 2006 ;8(6):434-41.

2. Mulu A, Kassu A, Anagaw B, Moges B, Gelaw A, AlemayehuM et al. Frequent detection of 'azole' resistant Candida species among late presenting AIDS patients in north west Ethiopia BMC Infectious Diseases 2013, 13:82.

3. Yapar N. Epidemiology and risk factors for invasive candidiasis. Therapeutics and clinical risk management. 2014; 10:95.The.

4. Sievert DM, Ricks P, Edwards JR, Schneider A, Patel J, Srinivasan A, Kallen A, Limbago B, Fridkin S. Antimicrobial-resistant pathogens associated with healthcare-associated infections summary of data reported to the National Healthcare Safety Network at the Centers for Disease Control and Prevention, 2009-2010. Infection Control \& Hospital Epidemiology. 2013;34(1):1

5. Zaoutis TE, Argon J, Chu J, Berlin JA, Walsh TJ, Feudtner C. The epidemiology and attributable outcomes of candidemia in adults and children hospitalized in the United States: a propensity analysis. Clinical infectious diseases. 2005;41(9):1232-9.

6. Zaragoza O, Mesa-Arango AC, Gómez-López A, Bernal-Martínez L, Rodríguez-Tudela JL, Cuenca-Estrella M. A process analysis of variables for standardization of antifungal susceptibility testing of nonfermentative yeasts. Antimicrobial agents and chemotherapy. 2011. 
7. Melhem MS, Bertoletti A, Lucca HR, Silva RB, Meneghin FA, Szeszs MW. Use of the VITEK 2 system to identify and test the antifungal susceptibility of clinically relevant yeast species. Brazilian Journal of Microbiology. 2013;44(4):1257-66.

8. Donay JL, Mathieu D, Fernandes P, Pregermain C, Bruel P, Wargnier A, Casin I, Weill FX, Lagrange PH, Herrmann JL. Evaluation of the automated Phoenix system for potential routine use in the clinical microbiology laboratory. Journal of clinical microbiology. 2004;42(4):1542-.

9. Bitew A, Abebaw Y. Vulvovaginal candidiasis: species distribution of Candida and their antifungal susceptibility pattern. BMC women's health. 2018;18(1):94.

10. Gunsilius E, Lass-Flörl C, Kähler CM, Gastl G, Petzer AL. Candida ciferrii, a new fluconazole-resistant yeast causing systemic mycosis in immunocompromised patients. Annals of hematology. 2001;80(3):178-9.

11. Liu W, Tan J, Sun J, Xu Z, Li M, Yang Q,etal.Inasive candidiasis in intensive care units in China: in vitro antifungal susceptibility in the China-SCAN study J Antimicrob Chemother. 2014; 69:162-

12. Pappas PG, Kauffman CA, Andes DR, Clancy CJ, Marr KA, Ostrosky-Zeichner L, etal .Clinical Practice Guideline for the Management of Candidiasis: 2016 Update by the Infectious Disease Society of America. Clin Infect Dis. 2016;62:409-17

13. Pfaller MA, Diekema DJ, Procop GW, Rinaldi MG. Multicenter comparison of the VITEK 2 antifungal susceptibility test with the CLSI broth microdilution reference method for testing amphotericin $B$, flucytosine, and voriconazole against Candida spp. Journal of clinical microbiology. 2007; 1;45(11):35228.

14. Sanguinetti M, Porta R, Sali M, La Sorda M, Pecorini G, Fadda G, Posteraro B. Evaluation of VITEK 2 and RapID yeast plus systems for yeast species identification: experience at a large clinical microbiology laboratory. Journal of clinical microbiology. 2007;45(4):1343-6.

15. Okonkwo E C, Alo MN, Nworie 0, Orji JO, Agah MV.Prevalence of oral candida albicans infection in HIVsero-positive patients in Abakaliki.American. Journal of Life Sciences. 2013; 1: $72-76$.

16. Spampinato C, Leonardi D. Candida infections, causes, targets, and resistance mechanisms: traditional and alternative antifungal agents. BioMed research international. 2013;2013

17. Banerjee P, Haider M, Trehan V, Mishra B, Thakur A, Dogra V, Loomba P. Cryptococcus laurentii fungemia. Indian journal of medical microbiology. 2013;31(1):75.

18. Vincent BM, Lancaster AK, Scherz-Shouval R, Whitesell L, Lindquist S. Fitness trade-offs restrict the evolution of resistance to amphotericin B. PLoS biology. 2013;11(10):e1001692.

19. Adjapong G, Bartlett $M$, Hale M, Garrill A. The isolation of Candida rugosa and Candida mesorugosa from clinical samples in Ghana. Sabouraudia. 2015;54(3):322-6. 
20. Colombo AL, Melo AS, Rosas RF, Salomão R, Briones M, Hollis RJ, Messer SA, Pfaller MA. Outbreak of Candida rugosa candidemia: an emerging pathogen that may be refractory to amphotericin $B$ therapy. Diagnostic microbiology and infectious disease. 2003;46(4):253-7

21. Minces LR, Ho KS, Veldkamp PJ, Clancy CJ. Candida rugosa: a distinctive emerging cause of candidaemia. A case report and review of the literature. Scandinavian journal of infectious diseases. 2009; 41(11-12):892-7.

22. You X, Li J, Li S, Shi W. Effects of lamellar keratectomy and intrastromal injection of $0.2 \%$ fluconazole on fungal keratitis. Journal of ophthalmology. 2015;2015

23. Moges B, Bitew A, Shewaamare A. Spectrum and the in vitro antifungal susceptibility pattern of yeast isolates in Ethiopian HIV patients with oropharyngeal candidiasis. International journal of microbiology. 2016; 2016.

24. Khawcharoenporn T, Apisarnthanarak A, Mundy LM. Non-neoformans cryptococcal infections: a systematic review. Infection. 2007;35(2):51.

25. Bernal-Martinez L, Gomez-Lopez A, Castelli MV, Mesa-Arango AC, Zaragoza O, Rodriguez-Tudela JL, Cuenca-Estrella M. Susceptibility profile of clinical isolates of non-Cryptococcus neoformans/nonCryptococcus gattii Cryptococcus species and literature review. Medical mycology. 2010;48(1):90-6

26. Smith N, Sehring M, Chambers J, Patel P. Perspectives on non-neoformans cryptococcal opportunistic infections. Journal of community hospital internal medicine perspectives. 2017;7(4):214-7.

27. Malini A, Oudeacoumar $P$, Udayashankar C. Onychomycosis due to Trichosporon mucoides. Indian Journal of Dermatology, Venereology, and Leprology. 2011;77(1):76.

28. Rizzitelli G, Guanziroli E, Moschin A, Sangalli R, Veraldi S. Onychomycosis caused by Trichosporon mucoides. International Journal of Infectious Diseases. 2016 ;42:61-3.

\section{Figures}




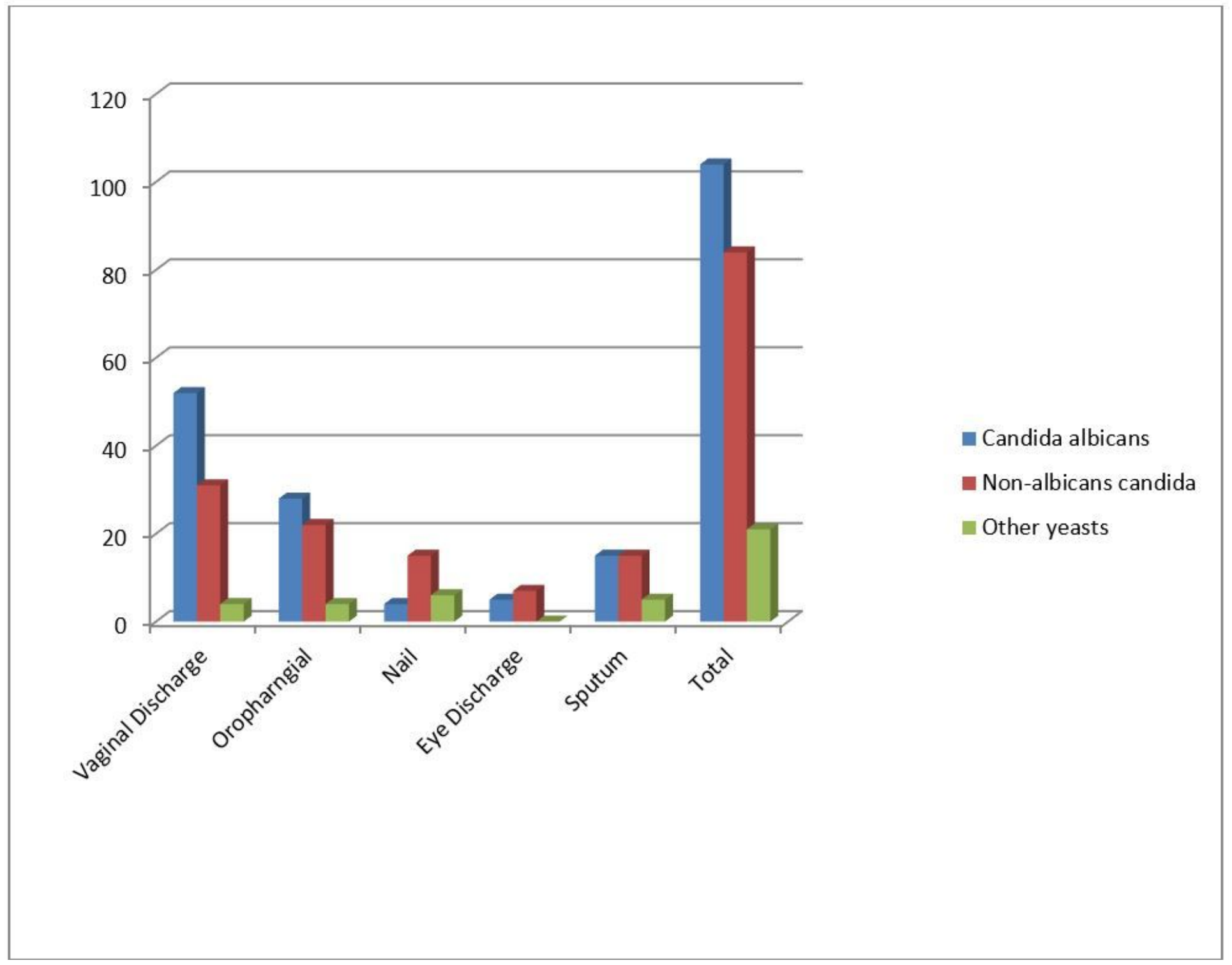

Figure 1

Frequency of Candida albican, non-albicans Candida and other yeasts species from different clinical samples at Arsho medical laboratory in Addis Ababa, Ethiopia from January, 2018 to September. 